Konferenz der Datenschutzbeauftragten des Bundes und der Länder

\title{
94. DSK am 08./09. November 2017 in Oldenburg
}

Unter dem Vorsitz der Landesbeauftragten für den Datenschutz Niedersachsen, Barbara Thiel, hat die Konferenz der unabhängigen Datenschutzbehörden des Bundes und der Länder (DSK) auf ihrer Herbsttagung am 8. und 9. November 2017 in Oldenburg aktuelle Themen des Datenschutzes diskutiert.

Unter der Überschrift „Keine anlasslose Vorratsspeicherung von Reisedaten" fordert die DSK die jeweils zuständigen Gesetzgeber auf, zeitnah und konsequent die Speicherung von Fluggastdaten (Passenger Name Records - PNR-Daten) im Sinne des EuGH-Gutachtens zum Fluggastdatenabkommen zwischen Kanada und der EU vom Juni 2017 nachzubessern. Dies gilt insbesondere für das deutsche Fluggastdatengesetz.

Zugleich fordert die DSK die Bundesregierung auf, sich auf europäischer Ebene für eine den Anforderungen der EU-Grundrechtecharta und der Rechtsprechung des EuGH entsprechende Ausgestaltung des angestrebten Entry-Exit-Systems (EES) sowie des EU-weiten Reiseinformations- und -genehmigungssystems (ETIAS) einzusetzen.

„Zwar hält der EuGH es grundsätzlich für zulässig, Fluggastdaten automatisiert zu übermitteln und auszuwerten, um Personen zu identifizieren, die eine potentielle Gefahr für die öffentliche Sicherheit darstellen und bei ihrer Einreise einer gewissenhaften Kontrolle unterzogen werden sollen“, erläutert die Vorsitzende. Allerdings haben die übermittelten Daten ihren Zweck erfüllt, wenn sich während des Aufenthaltes keine konkreten Anhaltspunkte für geplante terroristische oder andere schwere Straftaten ergeben haben. „In diesem Fall sieht der EuGH keine Rechtfertigung für eine weitere Speicherung der Daten“, betont Barbara Thiel.

Mit einer weiteren Entschließung fordert die Konferenz die Umsetzung der Datenschutzgrundverordnung auch im Medienrecht.

Anlass dafür sind die bisher durch die Länder vorgelegten Entwürfe für die Novellierung der Rundfunk-Staatsverträge. Diese schließen pauschal die Anwendbarkeit eines Großteils der Vorschriften der Datenschutz-Grundverordnung für den journalistischen Bereich aus und erklären lediglich drei Artikel für anwendbar. Dadurch wird nach Auffassung der DSK das durch
Art. 85 DSGVO vorgegebene Regel-Ausnahme-Verhältnis ins Gegenteil verkehrt.

„Die Vorschriften der Datenschutz-Grundverordnung sind grundsätzlich auch von Rundfunkanstalten und Presseunternehmen zu beachten. Der nationale Gesetzgeber ist lediglich befugt, begründete Ausnahmen zuzulassen, wenn diese erforderlich sind um das Recht auf Schutz personenbezogener Daten mit der Freiheit der Meinungsäußerung und der Informationsfreiheit in Einklang zu bringen", so Barbara Thiel. Eine faktische Beibehaltung der bisherigen nationalen Rechtslage würde dem nicht gerecht.

Gegenstand der Beratungen waren daneben Fragen der künftigen Einbindung der Datenschutzaufsichtsbehörden der Rundfunkanstalten und der Kirchen, die $\$ 18$ Absatz 1 Satz 4 BDSGneu zwingend vorschreibt, „sofern diese von der Angelegenheit betroffen sind“.

Auch die mögliche Fortsetzung des Dialogs mit Wirtschaftsunternehmen stand auf der Tagesordnung. Ein erstes Treffen im Sommer 2017 war seinerzeit von beiden Seiten positiv bewertet worden. Die DSK kam nun dem Ergebnis, dass ein solcher Austausch mit Wirtschaftsunternehmen und anderen Interessengruppen insbesondere zu Grundsatzfragen des Datenschutzes als sinnvoll anzusehen sei.

Dazu Barbara Thiel: „Um dem in der DSGVO normierten Auftrag zur Beratung, Sensibilisierung und Aufklärung gerecht werden zu können, halte ich einen Austausch zwischen Aufsichtsbehörden und Wirtschaftsunternehmen auf verschiedenen Ebenen für vorteilhaft und wünschenswert. Dabei muss es aus meiner Sicht vor allem um grundsätzliche datenschutzpolitische und datenschutzrechtliche Weichenstellungen gehen.

Eine solche gemeinsame Plattform auf strategischer Ebene kann aber auch die Basis für ein besseres und tiefergehendes Verständnis bilden, das allen Beteiligten zu gute kommen kann. Die Aufsichtsbehörden erhalten auf diese Weise aus erster Hand Einblicke und Informationen über datenschutzrelevante Entwicklungen innerhalb der Wirtschaft."

Den Wortlaut der Entschließungen finden Sie in der Rubrik Dokumentation in diesem Heft ab Seite 45. 